\title{
A Randomized Comparative Trial of the Knowledge Retention and Usage Conditions in Undergraduate Medical Students Using Podcasts and Blog Posts
}

\author{
Kelly Lien ${ }^{1}$, Alvin Chin ${ }^{2}$, Anton Helman ${ }^{3}$, Teresa M. Chan ${ }^{4}$ \\ 1. Michael G. Degroote School of Medicine, McMaster University 2. Faculty of Health Sciences, \\ Department of Medicine, Division of Emergency Medicine, McMaster University 3. Department of Family \\ and Community Medicine, University of Toronto 4. Emergency Medicine, McMaster University, \\ Hamilton, CAN
}

$\square$ Corresponding author: Kelly Lien, kelly.lien@medportal.ca

Disclosures can be found in Additional Information at the end of the article

\section{Abstract}

Introduction

Podcasts and blog posts have gained popularity in Free Open Access Medical education (FOAM). Previous work suggests that podcasts may be useful for knowledge acquisition in undergraduate medical education. However, there remains a paucity of research comparing the two mediums. This study aims to investigate if there are differences in knowledge acquisition and usage conditions by medical students using podcasts and blog posts.

Methods

Medical students were randomized to either the podcast or blog post group. They completed an initial online assessment of their baseline knowledge on the subject matter. Participants then received access to learning materials and were given four weeks to complete the follow-up assessment on their own time. Independent t-test, paired samples t-test, and a mixed ANOVA (analysis of variance) were conducted to assess knowledge acquisition. An intentionto-teach analysis was used to impute missing data from students lost to follow-up. Simple descriptive statistical data was used to describe media usage conditions.

Results

Received 11/13/2017

Review began 11/19/2017 Review ended 01/02/2018 Published 01/15/2018

๑) Copyright 2018

Lien et al. This is an open access article distributed under the terms of the Creative Commons Attribution License CC-BY 3.0., which permits unrestricted use, distribution, and reproduction in any medium, provided the original author and source are credited.
Completion of at least one follow-up assessment was comparable (68\% podcasts $(n=21 / 31)$, $73 \%$ blog posts $(n=22 / 30)$ ). Both groups showed significant improvements in their test scores, with an average $22 \%$ improvement for the podcast group and $29 \%$ for the blog post group. There was no significant statistical difference in knowledge acquisition between educational modalities overall. Students in the blog post group that completed both post-intervention quizzes showed a larger improvement than the podcast group in the toxicology topic, with similar improvements in the asthma topic. The podcast group tended to engage in multiple activities while using the learning materials (e.g. at least two to three of the following: driving, eating, chores, taking notes, exercising/walking), while the blog readers tended to do fewer activities (e.g. only one of the following: note taking, eating).

Conclusion 
This study suggests that podcasts and blog posts are useful for extracurricular knowledge acquisition by undergraduate medical students with no significant difference between the two modalities. The usage conditions for each type of media differ.

Categories: Emergency Medicine, Medical Education, Other

Keywords: podcasts, blog posts, medical education, asynchronous education, free medical online education, online learning resources, undergraduate medical education

\section{Introduction}

A core tenet of undergraduate medical education is teaching medical students to be selfdirected, lifelong learners [1]. Although medical schools often recommend a reading list of textbooks and primary literature, free open access medical education (FOAM) [2] has rapidly gained popularity with medical trainees, as this cohort increasingly relies on the internet to obtain up to date medical information [3-5]. FOAM encompasses a continually expanding database of resources for medical education, such as podcasts, blog posts, videos, and Twitter feeds.

Podcasts and blog posts are both increasingly being used in medical education [6-9]. Both are inexpensive to produce, easy to distribute, and offer great portability compared to traditional lectures. In addition, there is some evidence that these forms of media offer better learner engagement compared to traditional didactic lectures [5, 10-12]. Several residency programs, such as emergency medicine, are now incorporating newer teaching methods that include short interactive lectures, small group sessions and asynchronous components, such as blog posts and podcasts [13-15].

Although these resources are being incorporated by medical learners into their education, there is a paucity of research so far demonstrating their efficacy in learning outcomes. Many studies conducted in the literature so far lack a record of the knowledge gained as a measure of objective learning benefit [16-18] and, if it is recorded, combine podcasts and/or blog posts with further methods of teaching [11, 19-20]. Furthermore, these studies are often conducted with dental or nursing students rather than undergraduate medical students. A PubMed search of "medical education" AND "podcasts" found only two controlled trials that measured the effectiveness of podcasts as a tool for knowledge acquisition [10, 19]. Of note, both studies utilized a video podcast style uncommon with most FOAM resources. Schreiber and colleagues found no difference between live lectures versus video podcasts in terms of knowledge acquisition [19], while podcast users scored significantly higher in a post-test evaluation compared to text-based users in the study by Back and colleagues [10].

A recent prospective trial attempted to gain data on the prior utilization, usage conditions, and preferences of medical students regarding podcasts in undergraduate medical education [21]. The study found that students were listening to podcasts while engaging in other activities, such as driving, completing chores, and exercising. The secondary objective of determining knowledge acquisition and retention by students found that participants did experience a significant gain in knowledge overall.

This current study aimed to determine if there were differences in the knowledge acquisition by undergraduate medical students using either podcasts and blog posts. The secondary objective in this study was to obtain data on the extracurricular usage conditions of both types of media, including where and when students used the media, if they were participating in other activities simultaneously, how long they used it for, etc. 


\section{Materials And Methods}

\section{Participants}

Participants consisted of medical students from a Canadian medical school who did not participate in the study by Chin and colleagues [21]. They were recruited through the school's student mailing lists and the school's affiliated Facebook group. Students were informed that participation in this study would have no impact on their academic standing, and they submitted their informed consent for inclusion through an online consent form. Approval was gained from the Hamilton Integrated Research Ethics Board and the local protocol review committee of the university.

\section{Study design}

We used a randomized trial to compare two groups of learners. The first group was given information in podcast form, and the second group was provided the same information in blog post format. Participants were block-randomized by year of study and randomly assigned to either the podcast or blog post group. Both groups completed two sets of questionnaires: an initial questionnaire and pre-intervention multiple choice test, and a post-test and exit questionnaire. The pre-test questionnaire included questions on the student's familiarity with podcasts and blog posts, their current usage of the media, and their preference between the two, if applicable. The exit questionnaire included questions regarding the student's experience with the media type, whether they would consider using the media in the future, and what their preferences were in terms of time or word limits. Both groups were given four weeks to utilize the learning materials and to complete the final evaluation on their own time, which allowed for a measurement of extracurricular media usage patterns. The students were told to complete the exit questionnaire regardless of whether they used the learning materials to aid in the follow-up of participants, as well as to determine if there were any common reasons for not using the media.

The podcast group was provided with audio MP3 files on asthma (57:44 min) and toxicology (36:38 min), produced by AC, AH, and TC from a previous study at our centre [21]. The students were not aware of the learning topics when signing up for the study, only that the topics would be commonly encountered problems in medicine. The blog post group was provided with two website links to blog posts on the same topics, written by a medical student investigator (KL) and placed through the CanadiEM coached peer review process [22]. Blog posts were created based on the podcast content. The first post was entitled "Asthma in the ED" (emergency department) and contained 1,624 words and the second was entitled "A Practical Approach to Toxicology", which contained 1,196 words. The blog posts were written in English, and the podcasts were also recorded in English.

To determine knowledge acquisition, 11 multiple-choice questions were developed for the asthma topic, and 10 were developed for the toxicology topic. Quiz contents were directly extracted from the provided learning material. Two emergency staff physicians reviewed the questions and answers to determine content validity. The same questions were used for the preand post-intervention evaluations. The exit questionnaire included questions regarding the activities performed while using the media. Multiple-choice quizzes and questionnaires were offered using Google Forms (Google Inc., Mountain View, CA, USA).

\section{Analysis}

Paired-samples t-tests were performed to measure knowledge changes between pre- and postintervention for each of the two media types. An independent-samples t-test was used to determine if there was any significant difference between initial pre-intervention scores. We used a mixed analysis of variance (M-ANOVA) to compare the effect of media type on learning 
benefit, with the within-subjects factor as time, and the between-subjects factor as media modality. The topic (asthma, toxicology) was nested under modality. For students who were lost to follow-up, an "intention-to-teach" analysis was performed wherein an individual's original baseline knowledge score was used as their final quiz score. This was the most conservative method to account for missing data, with an assumption that students would not lose knowledge by using the learning materials and that knowledge would not decay over the length of the study. A separate analysis was conducted for the students that completed both post-intervention tests.

\section{Sample size calculation}

This trial was designed as a superiority trial with podcasts as the control arm to be compared to blog posts. Power calculations were performed a priori to determine the required sample size to detect a significant difference in knowledge acquisition between the two groups. As is conventional in the literature, an $\alpha$ of 0.05 and power of $80 \%$ were chosen. The mean standard deviation of $15 \%$, as reported by Chin and colleagues, was used as students from that study could be expected to be drawn from the same population as the current one [21]. An increase of $10 \%$ in knowledge acquisition was chosen for this study, based on the study by Back and colleagues, which noted an $8.8 \%$ difference between podcast and text users [10]. Using these numbers, a sample size of 58 participants was needed.

\section{Results}

Our study was conducted from October to December of 2016. Sixty-five medical students enrolled in the study and completed the pre-intervention quiz. First and second-year students were equally represented (33 and 28 students, respectively). Four third-year students participated. Thirty-three students were randomised to the podcast group and 32 to the blog post group (Figure 1). 


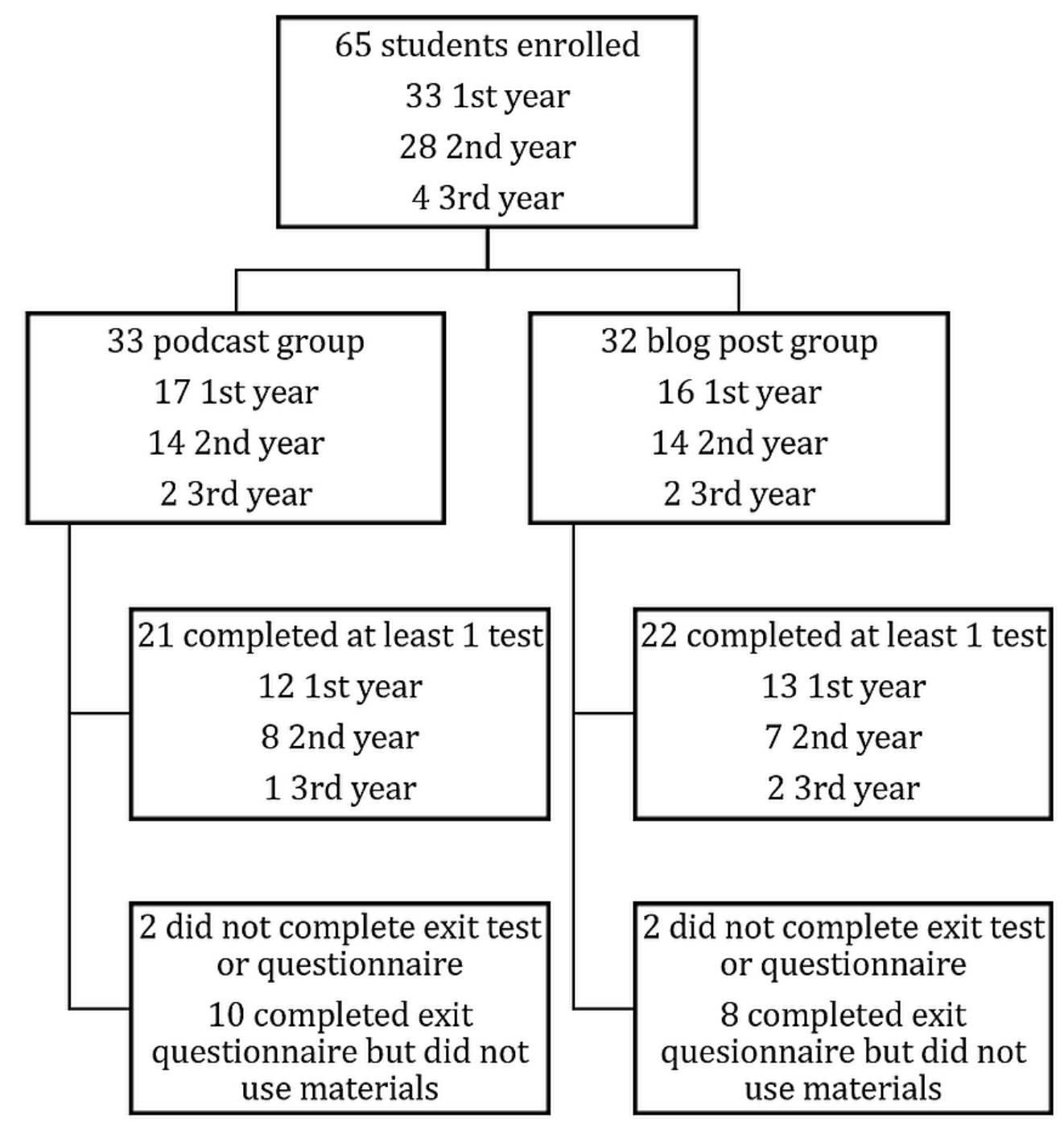

FIGURE 1: Study enrollment diagram

Second and third-year students were randomized to either group in equal numbers. Two students in each of the two arms did not complete the exit questionnaire and were lost to follow-up. Ten students $(32 \%, \mathrm{n}=31)$ in the podcast and eight $(25 \%, \mathrm{n}=32)$ in the blog post group completed the initial pre-intervention quiz but did not utilise the learning materials. These students completed the exit questionnaire stating that they had not used the media and were analysed using the "intention-to-teach" method as described. The scores from these students were imputed into the final calculations as described in the Methods section. Completion of at least one follow-up test was comparable (68\% podcasts, $73 \%$ blog posts).

\section{Learning outcomes}

Pre- and post-intervention scores using the intention-to-teach analysis are shown in Figure 2. 


\section{Cureus}

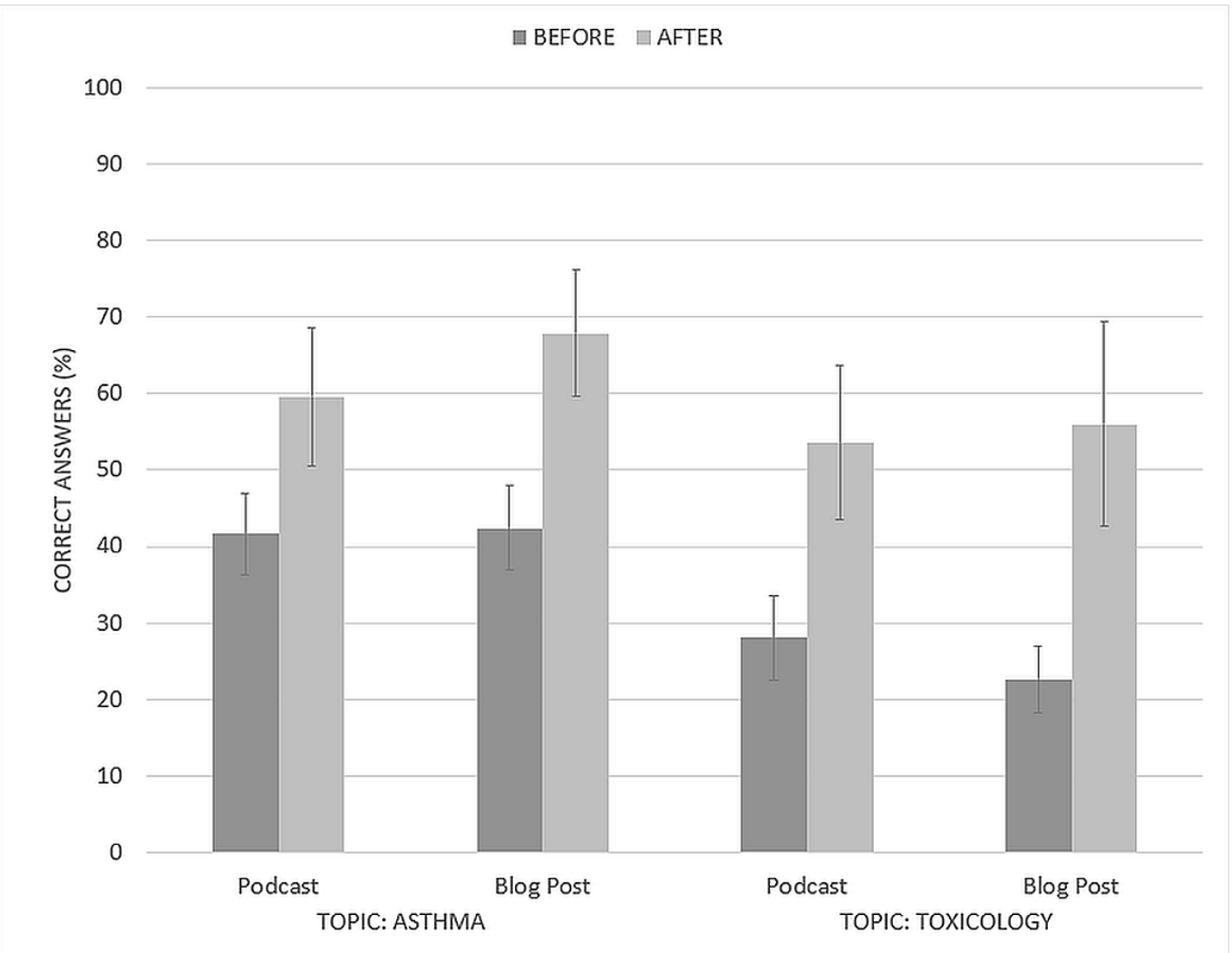

FIGURE 2: Quiz results for asthma and toxicology topics using intention-to-teach analysis

Mean multiple choice quiz results on asthma and toxicology topics before and after usage of podcasts or blog posts for learning by undergraduate medical students $(n=31$ for podcasts, $n=30$ for blog posts). Error bars represent the $95 \%$ confidence interval.

The mean pre-intervention asthma quiz score for both groups was $42 \%$ (95\% confidence interval (CI): $\pm 5.3 \%$ for podcasts, $\pm 5.6 \%$ for blog posts). For the toxicology topic, the pre-intervention quiz scores were $28 \%$ and $23 \%$ (95\% CI: $\pm 5.5 \%$ and $\pm 4.4 \%$ ) for the podcast and blog post groups, respectively. There was no significant difference in the pre-intervention scores between the groups (independent-samples t-test, Asthma: $p=0.84$; Toxicology: $p=0.13$ ). Both groups of students showed significant improvements in their test scores after using the learning materials. The podcast group improved by $18 \%$ and $26 \%$ for the Asthma and Toxicology topics, respectively (paired-samples t-test, $\mathrm{p}<0.01$ for both). Likewise, the blog post group improved by $25 \%$ and $33 \%$ (paired-samples t-test, $\mathrm{p}<0.01$ for both). However, there was no significant difference between the two modalities and no discernable interaction between the Topic and the Modality (M-ANOVA, Topic*Modality F(1,59) =0.001, p = 0.973).

The pre- and post-intervention scores for students who completed the post-intervention quizzes for both topics are shown in Figure $3(n=14$ and 11 for the podcast and blog post groups, respectively). 


\section{Cureus}

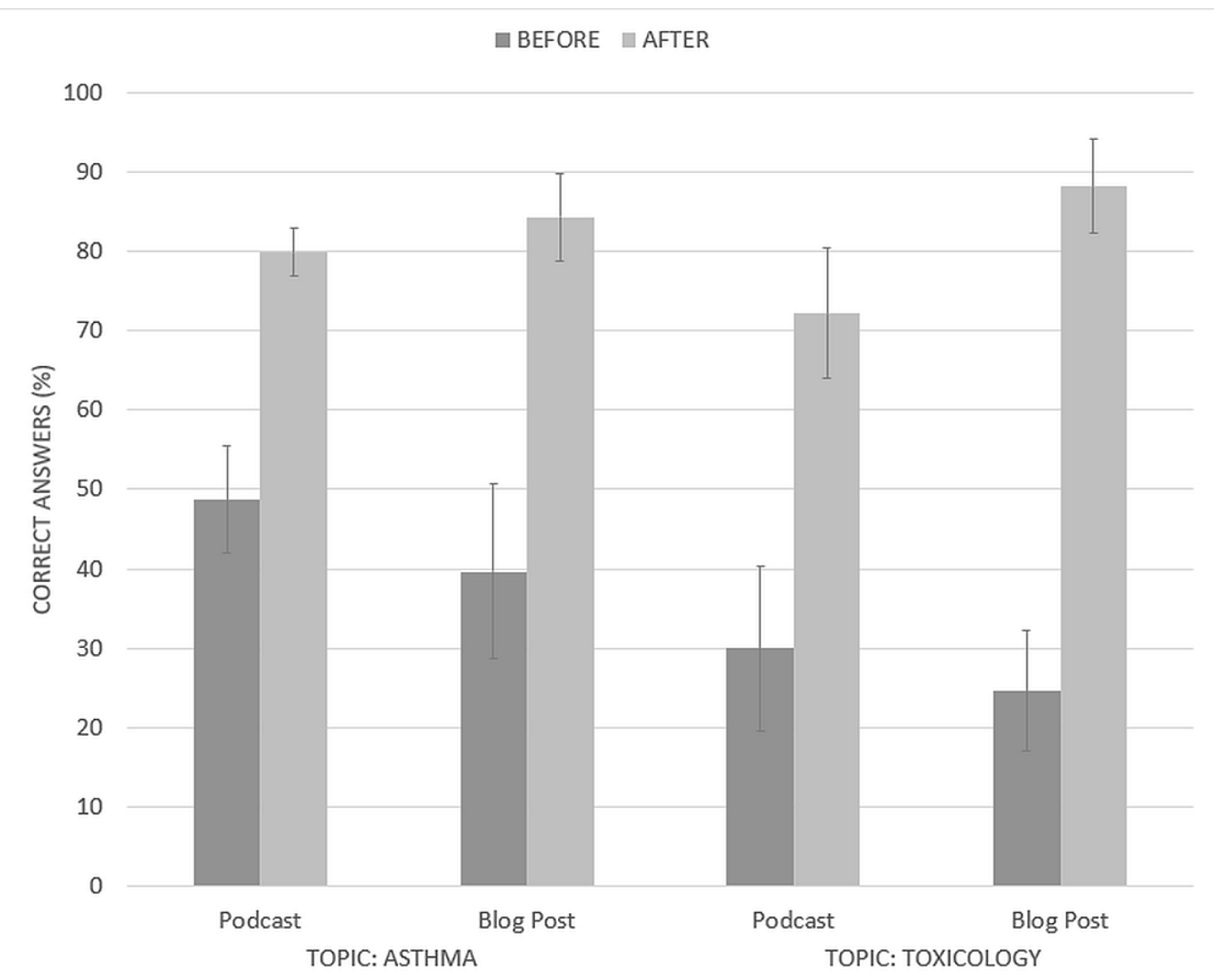

\section{FIGURE 3: Quiz results for students who completed both asthma and toxicology topics}

Mean multiple choice quiz results on asthma and toxicology topics before and after usage of podcasts or blog posts for learning by undergraduate medical students who completed both topics ( $n=14$ for podcasts, $n=11$ for blog posts). Error bars represent the $95 \%$ confidence interval.

There was no significant difference in the pre-intervention scores between the groups (Asthma: $p=0.14$; Toxicology: $p=0.36$ ). Both groups of students showed significant improvements in their test scores after using the learning materials. The podcast group improved by $31 \%$ and $42 \%$ for the asthma and toxicology topics, respectively (paired-samples t-test, $\mathrm{p}<0.01$ for both). Likewise, the blog post group improved by $45 \%$ and $64 \%$, respectively (paired-samples t-test, $p$ $<0.01$ for both). While the improvements in the asthma topic were similar, there was a statistically significantly greater improvement for the blog post group in the toxicology topic compared to the podcast group ( $\mathrm{p}<0.01)$.

\section{Media usage conditions and exit questionnaire responses}

The podcast group used computers and mobile devices equally for listening to the media. Podcast users tended to listen to the media in multiple sessions (60\%) that were 15-30 minutes in duration on average. Students liked that the podcast "taught us how to approach a clinical presentation and walked us through steps for differential and management", "was easy to listen to and kept a constant volume level", and "was good for consolidating information". In terms of points of improvement for the podcast, a few comments were made regarding the use of acronyms that were not explained fully. For podcast length, $71 \%$ of students who used the asthma podcast thought it should be shorter; individual free-text comments suggested that 30 minutes was preferable to most. On the other hand, only $39 \%$ of the toxicology podcast users wanted the podcast to be shorter. Multiple comments stated that the longer length was 


\section{Cureus}

appropriate, as toxicology was a new subject matter that was not reviewed in medical school. The students that did not listen to the podcasts cited lack of time and podcast length to be barriers to media usage.

The blog post group also used computers and mobile devices equally to browse the websites. They tended to read the blog post all at once (72\%) rather than in multiple sessions. Students reported taking 25 minutes to read either blog post (average 1,500 words). None of the students thought either blog post should be shorter in length, while a few believed the toxicology blog post could have been longer to provide more background information. This group also stated that toxicology was a new learning topic for them. The blog posts were "like shorter versions of textbooks, and much more directed to students", “good for copying into my own notes', and "how I wish my tutorials were laid out". Suggestions for improvement included more pictures and diagrams.

Usage patterns differed between media types. The podcast group tended to engage in multiple activities (i.e., listening as well as another activity) while using the learning materials (79\%; $\mathrm{n}=$ 22/28) (Figure 4).

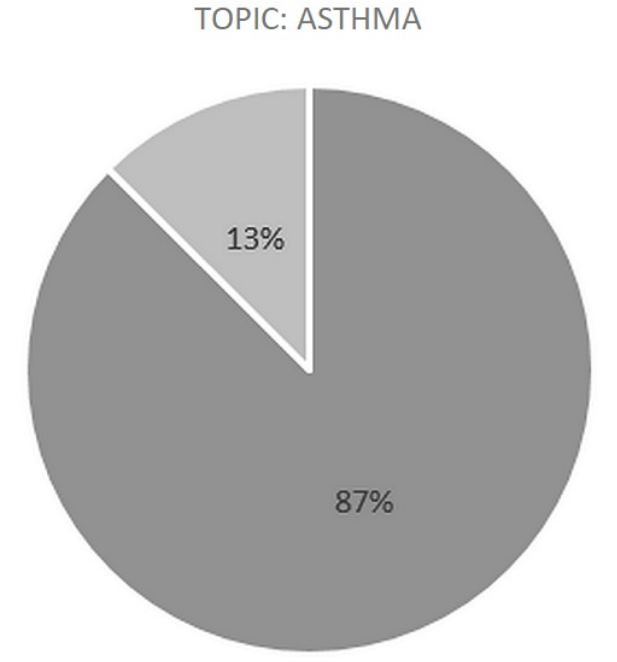

॥ LISTENING AND OTHER ACTIVITY*
TOPIC: TOXICOLOGY

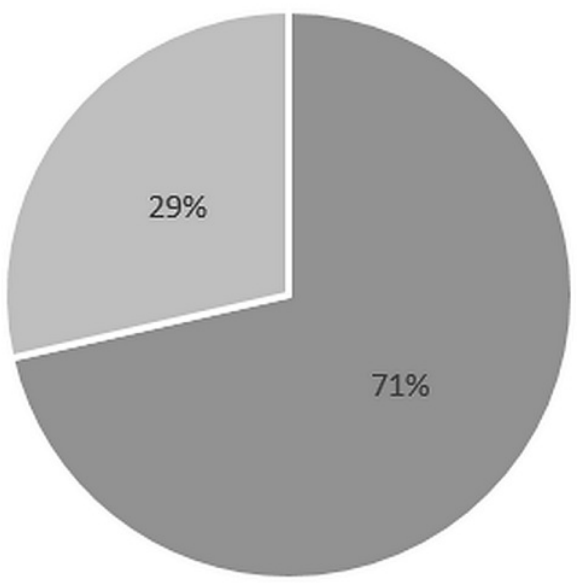

" ONLY LISTENING OR TAKING NOTES

\section{FIGURE 4: Podcast usage conditions by undergraduate medical students by topic}

Other activities include driving, physical activities, such as exercising, walking, chores, and eating.

The most common activities were driving, exercising, and eating. Of the two podcasts, students were more likely to only be listening or taking notes on the toxicology topic (29\% versus $13 \%$ ). Blog post users, on the other hand, only participated in multiple activities $31 \%$ of the time $(\mathrm{n}=$ 9/29) (Figure 5). 


\section{Cureus}

TOPIC: ASTHMA

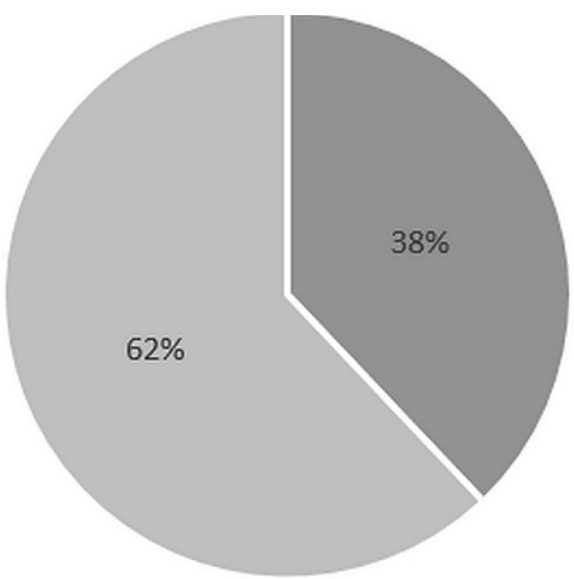

- READING AND OTHER ACTIVITY*
TOPIC: TOXIOCOLOGY

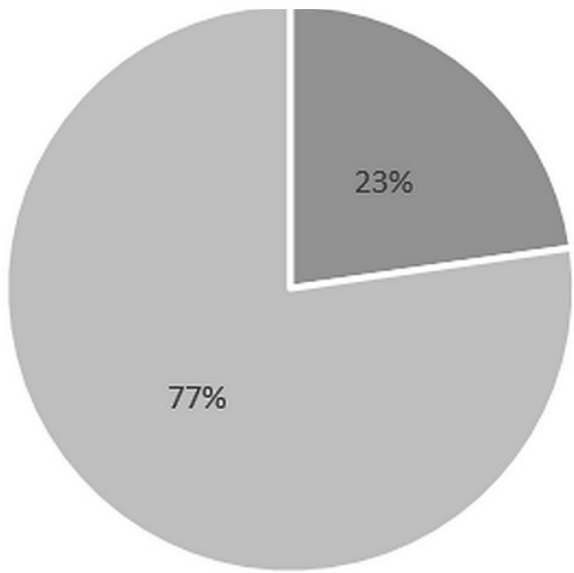

- ONLY READING OR TAKING NOTES

\section{FIGURE 5: Blog post usage conditions by undergraduate medical students by topic}

Other activities include physical activities, such as exercising, walking, and eating.

These activities included exercising, walking, and eating. The toxicology topic had more students only reading or taking notes compared to the asthma topic (77\% versus $62 \%$ ).

\section{Discussion}

FOAM resources, such as podcasts and blog posts, are increasingly being used by medical learners to supplement their learning [5, 8, 13-15]. It has been suggested that the "Digital Natives" [23] - students born after 1982 - even learn differently compared to previous generations, being more comfortable with audio and visual resources [3, 24]. Advantages of podcasts over traditional didactic lectures include ease of use, ability to learn on-the-go, and the means of reviewing and pausing at the user's leisure. In terms of blog posts, these are also easily accessible, portable, and easy for students to incorporate into existing notes. While FOAM resources have not yet replaced lectures and textbooks, studies suggest that they may aid in knowledge acquisition and retention in medical learners in combination with traditional resources $[10,18,20]$. Curricular developers in medical schools may consider curating suggested podcasts and blog posts for their students to complement existing didactics.

To the best of our knowledge, this is the first study that compares the two types of media to each other, as well as the first study to evaluate knowledge acquisition with blog posts. A review of the literature produces various publications with undergraduate medical students. These can be separated into studies evaluating podcasts alone for learning [17-18], studies with cross-over to lectures [11, 19], and the study by Back and colleagues comparing podcasts to traditional texts [10]. Of note, these studies generally used a podcast with visual components, e.g., PowerPoint presentations, while the current study used audio-only podcasts.

Both podcasts and blog posts improved knowledge on the two topics in our study. Interestingly, students in both groups were able to achieve a larger gain in knowledge with the toxicology topic as compared to asthma. According to the students, toxicology was a topic that was not 
covered directly in the undergraduate medical curriculum, whereas asthma was more familiar and covered in respiratory physiology lectures. This suggests that either podcast or blog post could be effective for learning a novel concept rather than merely for review of previously introduced material. While our study showed a similar improvement in test scores for the asthma topic, the blog post group enjoyed a statistically significant larger gain of knowledge for the toxicology topic when comparing students that completed both post-intervention tests. It may be that blog posts are better for learning a novel topic compared to podcasts, but further research is required.

Secondary objectives in the current study were to gain data on the extracurricular conditions in which students use these audio and text media. Literature suggests that multitasking (or more accurately, 'task switching') hinders learning [25-26]. In regards to audio-only media specifically, a study by Doolittle and Mariano found that students in a stationary position outperformed students in a mobile environment when using a portable digital media player [27]. However, Coens and colleagues conducted two studies with conflicting results regarding podcast usage and performing a secondary task while learning [28]. In the current study, even though students were more likely to be multitasking when listening to podcasts, their final post-intervention results were comparable to the blog post group. Additionally, while the blog post group was more diligent in taking notes while reviewing the material, this extra step did not appear to have a significant effect on knowledge acquisition. A possible explanation for this observation is that more students were exercising while listening to the podcasts compared to reading the blog posts, which may positively influence cognition as suggested in prior studies in the literature [29-30].

In terms of other considerations for podcast and blog post development, content creators should be conscious of the length of time required to use the resource. A podcast of 30 minutes or less or a blog post of 1,000 words is more acceptable to learners based on our study. Depending on the topic, users may be amenable to longer media when learning about a novel topic. "Approach to" types of topics may be the most popular with undergraduate medical students. Finally, to consolidate learning, multiple choice questions may be generated to promote retention of knowledge, in addition to a summary or handout sheet. These findings were similar to the conclusions of the study by Chin and colleagues [21].

Limitations to our study include using the same questions for both pre- and post-intervention tests. While students may have remembered the initial questions and were therefore primed for answering the exit quiz, we believe that this does not make a significant difference in our primary objective of determining if there was a difference in learning with the two media types, as both groups would experience the same priming effect. The results of this study were also based on a small number of medical students from a single medical school using two topics that are emergency medicine (EM)-based. Future studies should incorporate other disciplines since specialties, such as radiology and pathology, may not benefit from audio-based resources. Finally, as there was no control group in the study (i.e., a separate study arm that would consist of students without access to the learning materials), participants may have learned the topics in the medical school curriculum.

\section{Conclusions}

This study suggests that use of podcasts and blog posts by undergraduate medical students is useful for extracurricular knowledge acquisition. Students preferred podcasts with a duration of 30 minutes and blog posts consisting of 1,000 words or less. They also frequently multitask when listening to podcasts, while opting to write notes or only read when using blog posts. Other activities commonly performed when using these media were driving, exercising, doing chores, and eating. Content creators should consider these usage conditions when generating learning material and should include summary handouts and practice questions when possible. 


\section{Additional Information}

\section{Disclosures}

Human subjects: Consent was obtained by all participants in this study. Hamilton Integrated Research Ethics Board issued approval 14-686. Animal subjects: All authors have confirmed that this study did not involve animal subjects or tissue. Conflicts of interest: In compliance with the ICMJE uniform disclosure form, all authors declare the following: Payment/services info: A Medical Student Research Award from the Division of Emergency Medicine, Department of Medicine of McMaster University was awarded to Dr. Kelly Lien. Financial relationships: All authors have declared that they have no financial relationships at present or within the previous three years with any organizations that might have an interest in the submitted work. Other relationships: All authors have declared that there are no other relationships or activities that could appear to have influenced the submitted work.

\section{References}

1. Mann KV: Theoretical perspectives in medical education: Past experience and future possibilities. Med Educ. 2011, 45:60-68. 10.1111/j.1365-2923.2010.03757.x

2. Nickson CP, Cadogan MD: Free Open Access Medical education (FOAM) for the emergency physician. Emerg Med Australas. 2014, 26:76-83. 10.1111/1742-6723.12191

3. Sandars J, Morrison C: What is the Net Generation? The challenge for future medical education. Med Teach. 2007, 29:85-88. 10.1080/01421590601176380

4. Hart D, Joing S: The millennial generation and "the lecture”. Acad Emerg Med. 2011, 18:1186-87. 10.1111/j.1553-2712.2011.01215.x

5. Cheston CC, Flickinger TE, Chisolm MS: Social media use in medical education: a systematic review. Acad Med. 2013, 88:893-901. 10.1097/ACM.0b013e31828ffc23

6. Khogali SEO, Davies DA, Donnan PT, et al.: Integration of e-learning resources into a medical school curriculum. Med Teach. 2011, 33:311-18. 10.3109/0142159X.2011.540270

7. Paterson QS, Thoma B, Milne WK, et al.: A systematic review and qualitative analysis to determine quality indicators for health professions education blogs and podcasts. J Grad Med Educ. 2015, 7:549-54. 10.4300/JGME-D-14-00728.1

8. Maloney S, Chamberlain M, Morrison S, et al.: Health professional learner attitudes and use of digital learning resources. J Med Internet Res. 2013, 15:e7. 10.2196/jmir.2094

9. Gutmann J, Kühbeck F, Berberat PO, et al.: Use of learning media by undergraduate medical students in pharmacology: a prospective cohort study. PLoS One. 2015, 10:e0122624. 10.1371/journal.pone.0122624

10. Back DA, von Malotky J, Sostmann K, et al.: Superior gain in knowledge by podcasts versus text-based learning in teaching orthopedics: A randomized controlled trial. J Surg Educ. 2017, 74:154-60. 10.1016/j.jsurg.2016.07.008

11. Raupach T, Grefe C, Brown J, et al.: Moving knowledge acquisition from the lecture hall to the student home: A prospective intervention study. J Med Internet Res. 2015, 17:e223. 10.2196/jmir.3814

12. Vaccani JP, Javidnia H, Humphrey-Murto S: The effectiveness of webcast compared to live lectures as a teaching tool in medical school. Med Teach. 2016, 38:59-63. 10.3109/0142159X.2014.970990

13. Purdy E, Thoma B, Bednarczyk J, et al.: The use of free online educational resources by Canadian emergency medicine residents and program directors. CJEM. 2015, 17:101-106. 10.1017/cem.2014.73

14. Mallin M, Schlein S, Doctor S, et al.: A survey of the current utilization of asynchronous education among emergency medicine residents in the United States. Acad Med. 2014, 89:598-601. 10.1097/ACM.0000000000000170

15. Scott KR, Hsu CH, Johnson NJ, et al.: Integration of social media in emergency medicine residency curriculum. Ann Emerg Med. 2014, 64:396-404.

10.1016/j.annemergmed.2014.05.030

16. Shantikumar S: From lecture theatre to portable media: Students' perceptions of an enhanced podcast for revision. Med Teach. 2009, 31:535-38. 10.1080/01421590802365584 
17. White JS, Sharma N, Boora P: Surgery 101: Evaluating the use of podcasting in a general surgery clerkship. Med Teach. 2011, 33:941-43. 10.3109/0142159X.2011.588975

18. Narula N, Ahmed L, Rudkowski J: An evaluation of the “ 5 Minute Medicine” video podcast series compared to conventional medical resources for the internal medicine clerkship. Med Teach. 2012, 34:e751-55. 10.3109/0142159X.2012.689446

19. Schreiber BE, Fukuta J, Gordon F: Live lecture versus video podcast in undergraduate medical education: A randomised controlled trial. BMC Med Educ. 2010, 10:68. 10.1186/1472-6920-1068

20. Kalludi SN, Punja D, Pai KM, Dhar M: Efficacy and perceived utility of podcasts as a supplementary teaching aid among first-year dental students. Australas Med J. 2013, 6:45057.

21. Chin A, Helman A, Chan TM: P024: Extracurricular podcast use behaviour and effect on knowledge retention in undergraduate medical students. CJEM. 2016, 18:S86. 10.1017/cem.2016.200

22. Sidalak D, Purdy E, Luckett-Gatopoulos S, et al.: Coached peer review: developing the next generation of authors. Acad Med. 2017, 92:201-204. 10.1097/ACM.0000000000001224

23. Prensky M: Digital natives, digital immigrants part 1. On the Horizon. 2001, 9:1-6. 10.1108/10748120110424816

24. Oblinger DG, Oblinger JL, Lippincott JK: Educating the Net Generation. Oblinger DG, Oblinger JL (ed): Educause, Boulder, CO; 2005.

25. Ravizza SM, Hambrick DZ, Fenn KM: Non-academic internet use in the classroom is negatively related to classroom learning regardless of intellectual ability. Comput Educ. 2014, 78:109-14. 10.1016/j.compedu.2014.05.007

26. Sana F, Weston T, Cepeda NJ: Laptop multitasking hinders classroom learning for both users and nearby peers. Comput Educ. 2013, 62:24-31. 10.1016/j.compedu.2012.10.003

27. Doolittle PE, Mariano GJ: Working memory capacity and mobile multimedia learning environments: Individual differences in learning while mobile. J Educ Multimed Hypermedia. 2008, 17:511-30.

28. Coens J, Degryse E, Senecaut MP, et al.: Listening to an educational podcast while walking or jogging: Can students really multitask?. Int J Mob Blended Learn. 2011, 3:23-33. 10.4018/jmbl.2011070102

29. Schmidt-Kassow M, Kulka A, Gunter TC, et al.: Exercising during learning improves vocabulary acquisition: Behavioral and ERP evidence. Neurosci Lett. 2010, 482:40-44. 10.1016/j.neulet.2010.06.089

30. Hillman CH, Snook EM, Jerome GJ: Acute cardiovascular exercise and executive control function. Int J Psychophysiol. 2003, 48:307-14. 10.1016/S0167-8760(03)00080-1 